\title{
Mathematical Biology
}

National Cancer Institute

\section{Source}

National Cancer Institute. Mathematical Biology. NCI Thesaurus. Code C18867.

Interdisciplinary field of study in which theoretical, computational, or mathematical research is applied to or motivated by biology. 